\title{
Validation of a realistic, simple, and inexpensive EUS-FNA training model using isolated porcine stomach
}

Authors

Institution
Koki Hoshi, Atsushi Irisawa, Goro Shibukawa, Akane Yamabe, Mariko Fujisawa, Ryo Igarashi, Yoshitsugu Yoshida, Yoko Abe, Koh Imbe

Department of Gastroenterology, Aizu Medical Center, Fukushima Medical University, Aizuwakamatsu, Japan submitted 2. April 2016 accepted after revision 6. June 2016

\section{Bibliography}

DOI http://dx.doi.org/

10.1055/s-0042-110094

Published online: 5.8.2016

Endoscopy International Open 2016; 04: E1004-E1008

(c) Georg Thieme Verlag KG Stuttgart · New York

E-ISSN 2196-9736

\section{Corresponding author}

\section{Atsushi Irisawa}

Department of

Gastroenterology

Aizu Medical Center

Fukushima Medical University

21-2, Maeda, Tanisawa,

Kawahigashi, Aizuwakamatsu 969-3492

Japan

irisawa@fmu.ac.jp
Background and study aims: Trainees are required to learn EUS-FNA using a model before working with a patient. The aim of the current study was to validate a new training model developed for EUS-FNA.

Patients and methods: Several fresh chicken tenderloins were embedded as target lesions in the submucosal layer of an isolated porcine stomach. The stomach was fixed to a plate with nails, and was placed in a tub filled with water. The primary endpoint was feasibility of the newly developed model for EUS-FNA training, evaluated as follows: 1) visualization of the target lesion with blinding for lesion location; 2) penetrability of the needle; 3) sampling rate of macroscopic specimen; and 4) ROSE capability. Secondary endpoints were its durability and utility for multiple EUS-FNA procedures during EUS-FNA training, and the ease and cost of preparing the model.

\section{Introduction}

$\nabla$

Since the initial report about it 1992, endoscopic ultrasound-guided fine-needle aspiration (EUSFNA) has been incorporated into the diagnostic and staging algorithm for evaluation of benign and malignant diseases of the gastrointestinal tract and of adjacent organs. Expertise in EUS requires both cognitive and technical proficiency $[1,2]$. With the expanding applications of EUSFNA, procedural difficulties together with the risk of complications have concomitantly increased. Therefore, trainees are required to learn EUS-FNA using a model before working with a patient. In fact, European Society of Gastrointestinal Endoscopy (ESGE) guidelines recommend discouraging trainees from performing EUS-FNA with self-learning [3] as it is critical to perform procedures safely with certainty.

The ESGE guidelines for EUS-FNA recommend a combination of the use of different simulators, and, if available, live swine during EUS-FNA train-
Results: Six endoscopists ( 1 expert, 5 trainees) attempted EUS-FNA procedures using this model. The target lesion could be identified clearly, and EUS-FNA could be performed with realistic resistance felt. In addition, rapid on-site evaluation could be easily achieved. Based on 10 needlings by each endoscopist, adequate specimens for histology could be macroscopically taken with an average $85 \%$ success rate. Visibility and maneuverability were maintained throughout all needlings. Preparation time for this model was less than 30 minutes with a total cost of $\$ 22$.

Conclusions: An easy-to-use and inexpensive training model with a realistic feel of needling was created. This model can potentially enable beginners to practice safe and effective EUS-FNA procedures. ing. A live swine is apparently the best model because of its anatomical resemblance to a human. Moreover, EUS-FNA competency using the model can be assessed by rapid on-site evaluation (ROSE). However, live animal models are cumbersome and costly. Training models using agar gel with fruits and vegetables are easily and cheaply produced but unrealistic. Moreover, adequate materials cannot be confirmed through pathological examination. We aimed to develop, without the use of a live animal, a realistic EUS-FNA training model for which ROSE is possible. The aim of the current study was to validate a new training model developed for EUS-FNA.

\section{Patients and methods \\ $\nabla$}

\section{Study design}

This was a prospective trial for EUS-FNA training using a proposed new model produced from isolated porcine stomach. The primary endpoint 


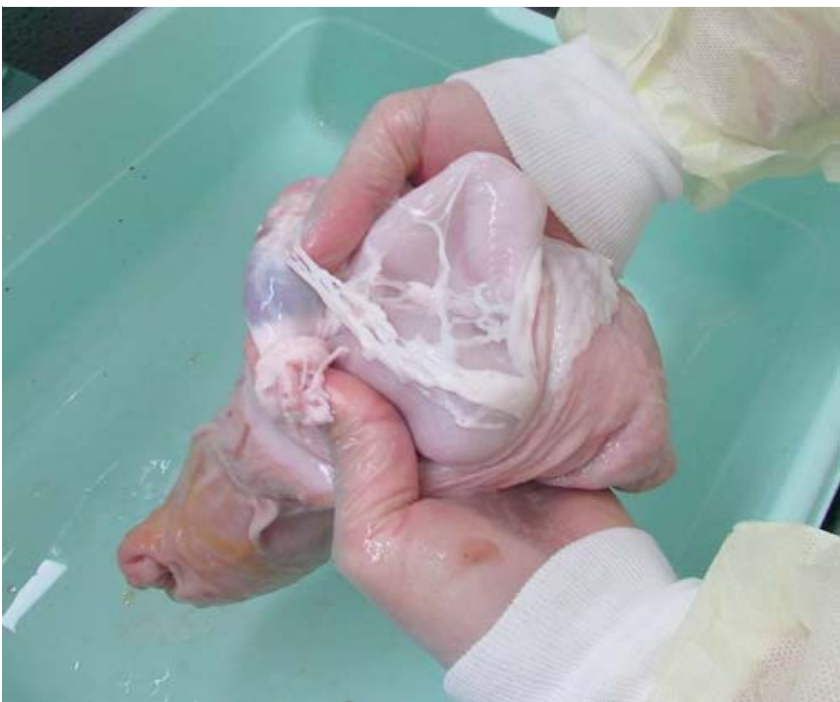

Fig. 1 The mucosal and serosal sides of the porcine stomach were reversed and the organ then was carefully washed.

was feasibility of the newly developed model for EUS-FNA training, evaluated as follows: 1) visualization of the target lesion with blinding for lesion location; 2) penetrability of the needle; 3 ) sampling rate of macroscopic specimen; and 4) ROSE capability. Secondary endpoints were its durability and utility for multiple EUS-FNA procedures during EUS-FNA training, and the ease and cost of preparing the model. The model was evaluated by 6 endoscopists ( 1 expert who had performed EUS-FNA for more than 500 patients and 5 trainees who had performed EUS-FNA on 10 to 20 patients).

\section{Preparation of the EUS-FNA training model}

This experimental study was performed at a room in our institution between January and July of 2015. The EUS-FNA training model using the isolated porcine stomach was created as follows. First the mucosal and serosa sides of the porcine stomach were reversed ( $\bullet$ Fig. 1). Next 3 fresh chicken tenderloins $(20-30 \mathrm{~mm}$ diameter) were embedded in the submucosal layer through incision of the mucosa for presentation as target lesions for EUS-FNA $($ Fig.2). Then the incision was sutured using silk thread and the stomach was re-reversed. Finally the stomach was fixed onto a plate with nails and placed in a tub filled with water. Two models for were made for the 6 endoscopists with 1 target lesion for each endoscopist.

\section{Equipment}

A curved-linear echoendoscope with an ultrasound processor (GF-UC240P and EU-ME1; Olympus Medical Systems Corp., Tokyo, Japan) was used with 22-gauge needles (EZ-shot 22G; Olympus Medical Systems Corp., Tokyo, Japan). In addition, ROSE was performed using modified Giemsa staining (Cyto Quick; Muto Pure Chemicals Co., Tokyo, Japan).

\section{EUS-FNA and ROSE}

The needle was passed through the biopsy channel of the echoendoscope and advanced through the gastric mucosa under EUS guidance into the target lesion with visualization of the needle in real time. After guidance into the target lesion, the stylet was removed and the needle was moved back and forth 10 times within the mass, while suction was being applied using a $20-\mathrm{mL}$

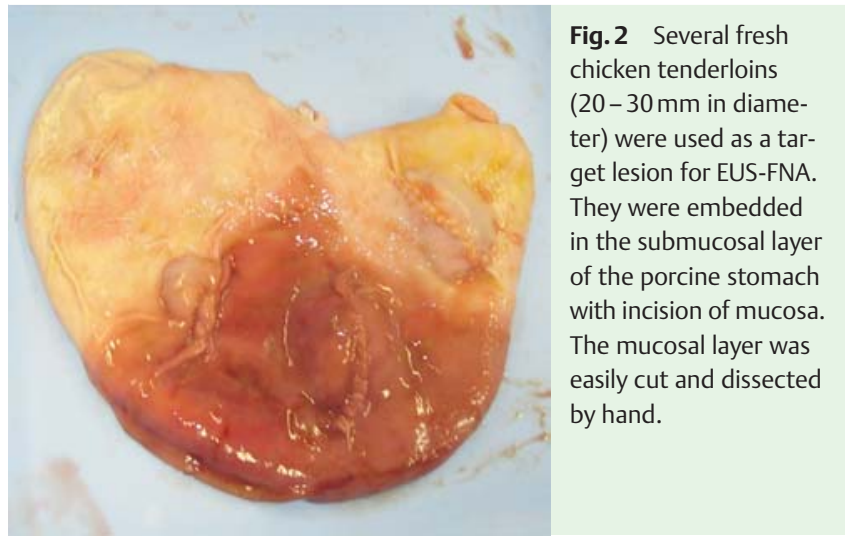

syringe. Subsequently, the suction syringe was released and the needle was withdrawn into the catheter and removed. The aspirated materials were sprayed onto glass slides by expulsion using a syringe or by stylet, and air-dried with a dryer for ROSE. The cell samples were stained with Cyto-Quick stain on glass slides.

\section{Evaluation of utility value}

For evaluation of the model's feasibility, multiple use, and durability, 6 endoscopists attempted EUS-FNA procedures using this model. Visualization of the target lesion (chicken tenderloins) was rated by each endoscopist using 3 categories: good, admissible, fail. The realism of needle penetrability in comparison with needling for pancreatic solid mass without necrosis was rated by each endoscopist using 4 scoring categories: realistic feel ( 4 points), almost realistic feel (3 points), a little different from a realistic feel ( 2 points), very different from a realistic feel ( 1 points). For this evaluation, 3 needlings were performed by each endoscopist and the mean value was calculated. Obtainment of a macroscopic specimen was macroscopically evaluated with an agreement by 2 endoscopists. ROSE capability was evaluated based on the ease of ROSE performance on adequate samples. ROSE was performed and evaluated by the endoscopist according to previous literature [4].

Each endoscopist performed 10 needlings for the assigned target lesion to assess model durability and its use for multiple procedures. Results were assessed based on obtaining of the macroscopic specimen (the grossly-visible core sample for histology) and the performing ROSE.

The ease and cost of model preparation were evaluated as preparation time and total cost of making the model.

\section{Results}

$\nabla$

\section{Feasibility}

Six endoscopists ( 1 expert, 5 trainees) attempted EUS-FNA procedures using this model. The target lesions (chicken tenderloins) were clearly identified in all cases ( $\bullet$ Fig.3). In addition, the silk thread which was used for the embedding of the target lesion in the submucosal layer did not produce ultrasound artifacts. All endoscopists evaluated the target lesions as "good visualization" in this model. All endoscopists were able to perform EUS-FNA using an FNA needle ( $\bullet$ Fig. 4 ). Mean score of the realism of the needle penetrability was $3.89 \pm 0.32($ mean \pm SD) points ( Table 1 ). ROSE was easily performed in all cases where an adequate sample was obtained ( $\bullet$ Fig.5). 


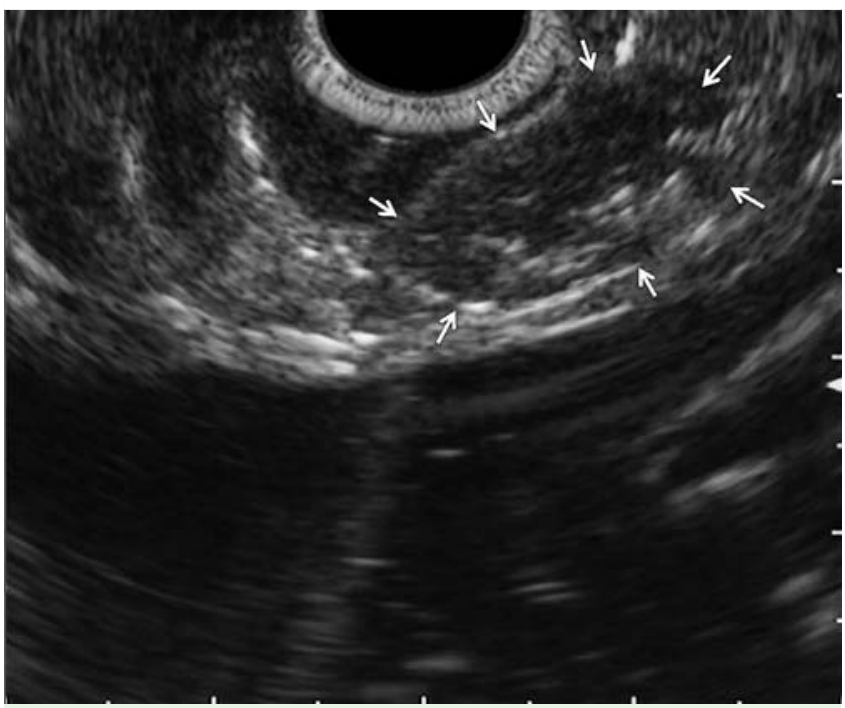

Fig. 3 Target lesion (chicken tenderloins) was clearly identifiable (arrows). It was visualized as a hypoechoic mass similar to a subepithelial lesion.

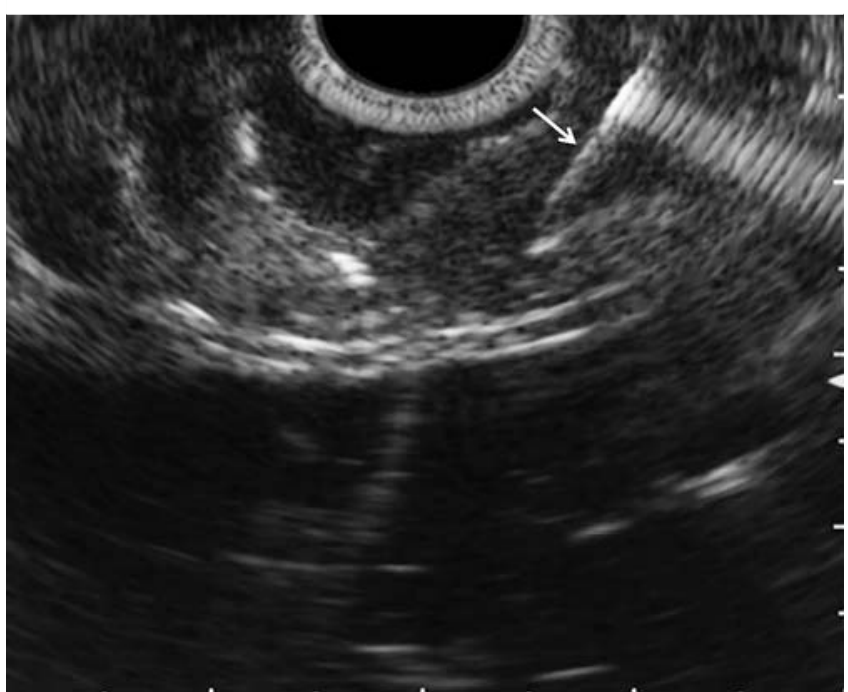

Fig. 4 Performing EUS-FNA on the model using an FNA needle resulted in a feeling of resistance. The FNA needle was visualized clearly (arrow), and visibility and maneuverability were maintained until the last needling.

\begin{tabular}{|llll|}
\hline & 1st approach & 2nd approach & 3 rd approach \\
\hline Expert & 4 & 4 & 4 \\
\hline Trainee A & 4 & 4 & 4 \\
\hline Trainee B & 4 & 4 & 4 \\
\hline Trainee C & 3 & 3 & 4 \\
\hline Trainee D & 4 & 4 & 4 \\
\hline Trainee E & 4 & 4 & 4 \\
\hline
\end{tabular}

4: realistic feel; 3: almost realistic feel; 2: a little different from a realistic feel; 1 : very different from a realistic feel

\section{Repeatability and durability}

In 10 needlings by each endoscopist, macroscopic specimens for histology were macroscopically taken at an average $85 \%$ success rate $(8.5 \pm 0.84$ (mean \pm SD), range $8-10$ : 8 by experts, 8 by trainee $A, 8$ by trainee $B, 8$ by trainee C, 9 by trainee $D$, and 10 by trainee $\mathrm{E}$ ). Adequate samples for cytology produced results similar to those for histology.

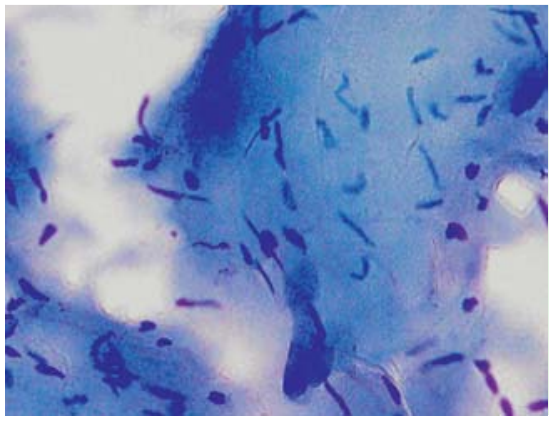

Fig. 5 Material obtained with EUS-FNA was immediately evaluated. Microscopic findings showed spindle cells.

EUS-FNA was performed 30 times using the same model. Visibility and maneuverability were maintained throughout all needlings in each model.

\section{Ease and cost of model preparation}

Reversal of the mucosal and serosa sides, and embedment of the chicken tenderloin in the submucosal layer did not pose difficulty. Preparation time was $28 \pm 2.83 \mathrm{~min}$ (mean \pm SD, range was 26 30 ) and total cost was $\$ 22.00$. All endoscopists could insert an echoendoscope into the stomach through a cutting plane of the esophagus.

\section{Discussion \\ $\nabla$}

EUS-FNA has become popular in recent years, and occupies an important position as a diagnostic intervention with various procedures for therapeutic interventional EUS having been developed. This development has necessitated more advanced techniques in EUS-FNA procedures, while beginners have also begun to perform the procedure more often. Although EUS-FNA is recognized as a safe procedure with a complication rate of approximately $1 \%$, it is possible for severe complications to occur, such as perforation, bleeding, and acute pancreatitis. Moreover, the endosonographers require the use of the high diagnostic capability of EUS-FNA, even if the operator is a beginner. Therefore, appropriate training is necessary to perform the procedure safely with certainty. ESGE technical guidelines discuss issues related to the learning curve, techniques, and complications of EUS-guided sampling, and the processing of specimens obtained with EUSFNA [5].

Saker et al. described that substantial variation exists in the speed of learning of all endoscopic procedures [6]. Today, materials available for learning EUS-FNA include common didactic materials (e.g., books, videos), simulators of various types, and live swine. Unfortunately, formal training programs are scarce even in western countries, and are more so in countries where the use of EUS-FNA is most developed. Nevertheless endoscopists learning EUS-FNA must practice with non-patient subjects before leaving clinical practice, and adequate training programs for endoscopists are few [7-9]. It is therefore necessary to provide all beginners with more efficient training.

"Hands-on" training methods for EUS-FNA have heretofore been divided into 4 categories: use of a tabletop model (EUS-FNA phantom with emplaced vegetables, fruits, or silicone blocks as targets in the agar gel); computer-based simulators; life-like simulators; and live animals [10]. Each model presents benefits and shortcomings. The animal models are used as virtual reality simulators for the training of both basic and advanced endoscopic techniques. To date, the usefulness of a live animal model 
for EUS-FNA has been reported [7,11]. The ESGE technical guidelines recommend a combination of the use of different simulators, and if available, live swine during EUS-FNA training. In fact, among the models available for "hands-on" training, live swine are the most realistic and can support the improvement of EUSFNA skills. In addition, ROSE can be performed using this model, which will provide a sense of fulfillment in EUS-FNA. Unfortunately, training using live swine is not practical for widespread use because of its expense and difficulty of preparation.

Other EUS-FNA models use agar gel with incorporated vegetables, fruits, or silicone blocks. Excised porcine stomach with grapes has been used for the practice of endoscope and needle handling. The salient benefits of this model are that it is simple and easy. Moreover, it can be stored and transported conveniently. However, needling using these models is overly simplified and cannot replicate the feeling of resistance that is associated with the organ [12]. In addition, in these models, mere pathological examination by ROSE cannot confirm the adequacy of the materials. Matsuda et al. produced a life-like simulator training model using the upper gastrointestinal tract from swine [10,13]. It consists of a specially made silicon case housing the ex-vivo swine stomach surrounded by a gelatin medium to provide acoustic coupling. Grapes are embedded in gelatin to simulate lymph nodes. The whole preparation is labor intensive, taking about 6 hours, excluding the swine organ preparation. The model can last 2 to 3 days in the refrigerator after use. During that time, the model can generate real-time EUS images of tissue and is the most realistic simulator of EUS-FNA aside from the live swine model. Although this model is a more realistic simulator than the EUS phantom, its preparation time is somewhat long.

Each model described above entails some shortcomings (unrealistic, unable to perform ROSE, expensive, long preparation time, etc.). Therefore our new EUS-FNA training model was created with the aim of resolving these difficulties. The basic concept of this model is based on the use of swine upper gastrointestinal tract and chicken tenderloin as a target lesion for realism. In fact, the penetrability of the needle closely approximates the sensation encountered in clinical practice. In addition, because chicken tenderloin was used for the target lesion, ROSE could be performed to confirm adequacy. Moreover, our model can be created easily at a low cost of about $\$ 20$ in approximately 30 minutes.

In the production of this model, the various organs/foodstuffs (liver, kidney, testes, ovaries, eye, and tenderloin) extracted from a swine/chicken were tested to ascertain organ suitability for use as target lesions. Several factors including ease of placement in the submucosal layer, visibility on EUS, feasibility of specimen collection and performance of ROSE were used as criteria. Chicken tenderloin was chosen for use as a target lesion. The same target lesion was punctured over 30 times, with good visualization of the target lesion (chicken tenderloin) throughout. Therefore, chicken tenderloin is a durable material for use as an EUS-FNA training model.

In addition, similar results rates of approximately $70 \%$ to $80 \%$, were found for both trainees and expert study participants. It is particularly interesting that this result is similar to the sampling rate of EUS-FNA for gastrointestinal subepithelial lesions. Our training model is not overly easy but remains achievable. From this perspective, this is a suitable EUS-FNA training model. However, because animal tissue is used in this model, a dedicated echo-endoscope may be necessary.
Although this new model is simple and inexpensive, it has some limitations compared to a realistic EUS-FNA model. First, the critical positioning phase before the EUS-FNA is more easily performed in this model. Second, the risks and the difficulties in vascular interpositioning with the target during EUS-FNA cannot be learned due to the lack of vascular structures in this model. Third, the penetrability of the needle during the EUS-FNA could be different from the live model due to the absence of backpressure from adjacent organs. Lastly, although management of needle passage through the gastric wall is interesting to anticipate and to appreciate during the hands-on session, it is a primary use of this model. However, the basic technique of EUS-FNA with a realistic feel can be learned from this model and ROSE can be performed to confirm material adequacy.

In this study, we have created a training model for EUS-FNA with possible cytological and histological assessment of samples. It was recently reported that acquisition of a large amount of tissue and a histological diagnosis were required for a higher rate of accuracy in pathological diagnosis $[14,15]$, and various needle devices and techniques for tissue acquisition therefore were developed. Thus, this model will potentially contribute not only to training but also to development and examination of such devices and techniques. Moreover, models for other interventional EUS, such as cyst drainage, can be produced through modifications to the current model (using a gallbladder or bladder cyst). The model described herein might also be applicable for training and development of various therapeutic uses of EUS.

\section{Conclusions}

\section{$\nabla$}

In conclusion, we have created a training model that is easy and inexpensive to use, offering a realistic penetrability of the needle. The greatest benefit of our model is that it allows confirmation of adequate specimen obtainment. This model will enable beginners to practice and develop safe and effective EUS-FNA procedures.

\section{Competing interests: None}

\section{References}

1 Henriksen FW, Hancke S. Percutaneous cystogastrostomy for chronic pancreatic pseudocyst. Br J Surg 1994; 81: 1525-1528

2 Erickson RA. EUS-guided FNA. Gastrointest Endosc 2004; 60: 267 - 279

3 Polkowski M, Larghi A, Weynand B et al. Learning, techniques, and complications of endoscopic ultrasound (EUS)-guided sampling in gastroenterology. European Society of Gastrointestinal Endoscopy (ESGE) Technical Guideline. Endoscopy 2012; 44: 190-206

4 Hikichi T, Irisawa A, Bhutani MS et al. Endoscopic ultrasound-guided fine-needle aspiration of solid pancreatic masses with rapid on-site cytological evaluation by endosonographers without attendance of cytopathologists. J Gastroenterol 2009; 44: 322 - 328

5 Dumonceau JM, Polkowski M, Larghi A et al. Indications, results, and clinical impact of endoscopic ultrasound (EUS)-guided sampling in gastroenterology: European Society of Gastrointestinal Endoscopy (ESGE) Clinical Guideline. Endoscopy 2011; 43: 897-912

6 Sarker SK, Albrani T, Zaman A et al. Procedural performance in gastrointestinal endoscopy: live and simulated. World J Surg 2010; 34: $1764-1770$

7 Barthet M, Gasmi M, Boustière C et al. EUS training in a live pig model does it improve echo endoscope hands-on and trainee competence? Endoscopy 2007; 39: 535-539

8 Rösch T. State-of-the-art lecture: endoscopic ultrasonography: training and competence. Endoscopy 2006; 38: 69-72

9 Savides TJ, Fisher AH, Gress FG et al. 1999 ASGE endoscopic ultrasound survey. Gastrointest Endosc 2000; 52: 745 - 750 
10 Matsuda K, Hawes $R H$, Sahai $A V$ et al. The role of simulators, models, phantoms. Where's the evidence? Endoscopy 2006; 38: 61-64

11 Bhutani MS, Hoffman BJ, Hawes RH. A swine model for teaching endoscopic ultrasound (EUS) imaging and intervention under EUS guidance. Endoscopy 1998; 30: 605-609

12 Fritscher-Ravens A. Training model for Endoscopic Ultrasound-gided Fine-Needle Aspiration of Lymph Nodes. Gastroenterol Hepatol (N.Y.) 2013; 9: 380-382

13 Matsuda K, Tajiri H, Hawes RH. How shall we experience EUS and EUSFNA before the first procedure? The development of learning tools Dig Endosc 2004; 16: 236 - 239
14 Yasuda I, Goto N, Tsurumi $H$ et al. Endoscopic ultrasound guided fine needle aspiration biopsy for diagnosis of lymphoproliferative disorders: feasibility of immunohistological, flow cytometric, and cytogenetic assessments. Am J Gastroenterol 2012; 107: 397-404

15 Itoi $T$, Tsuchiya $T$, Itokawa $F$ et al. Histological diagnosis by EUS-guided fine-needle aspiration biopsy in pancreatic solid masses without onsite cytopathologist: a single-center experience. Dig Endosc 2011; 23: $34-38$ 